\title{
Double Sheaths in RF Discharges
}

\author{
Luís Marques, Gérard Gousset, and Luís L. Alves
}

\begin{abstract}
The paper analyses the formation of double space-charge sheaths, associated to the development of Double Ionization Structures in Radio-Frequency discharges. A simulation tool is used to generate space-time images of the ionization rate in hydrogen and in helium, obtained by inducing artificial modifications in the mobility of charged particles, with these gases.
\end{abstract}

Manuscript received July $1^{\text {st }}, 2004$; revised November $23^{\text {rd }}, 2004$

L. Marques and L.L. Alves are with the Centro de Física de Plasmas, Instituto Superior Técnico, 1049-001 Lisboa, Portugal (e-mail: llalves@alfa.ist.utl.pt).

L. Marques is also with the Departamento de Física, Universidade do Minho, 4710-057 Braga, Portugal.

G. Gousset is with the Laboratoire de Physique des Gaz et des Plasmas, Université de Paris-Sud, 91405 Orsay Cedex, France. 
The development of Double Ionization Structures (DIS) in Capacitively Coupled RadioFrequency (CCRF) discharges has been reported in various works, either experimental (e.g., space-time resolved measurements of the 3p-2s Balmer-alpha excitation transition, at wavelength $653.3 \mathrm{~nm}$ [1]) or numerical simulation (fluid or Particle-in-Cell/Monte-Carlo models for hydrogen RF discharges [2]-[3]). These structures appear in near-electrode regions, thus being often called as double sheaths, and its formation is sometimes associated to the presence of high-mobility ions [2]. This explanation is, however, incomplete.

First, the well-known fact that CCRF discharges produced in hydrogen feature the formation of DIS, whereas these structures are not present for discharges produced in helium, cannot be explained on the mere basis of ion mobility differences. In fact, the mobilities of $\mathrm{H}_{3}^{+}$ions (the most abundant hydrogen ions) and $\mathrm{He}^{+}$ions (at $300 \mathrm{~K}$ gas temperature) are practically equal: $\mu\left(\mathrm{H}_{3}^{+}\right)=10.3 \mathrm{~V}^{-1} \mathrm{~s}^{-1} \mathrm{~cm}^{2}$ and $\mu\left(\mathrm{He}^{+}\right)=12.1 \mathrm{~V}^{-1} \mathrm{~s}^{-1} \mathrm{~cm}^{2}$ [4]. Incidentally, electrons have a mobility twice as higher in helium than in hydrogen [or, equivalently, the electron-neutral momentum transfer cross-section in helium $\sigma_{c}(\mathrm{He})$ is approximately a factor of two bellow that of hydrogen $\sigma_{c}\left(\mathrm{H}_{2}\right)$ ]. Second, CCRF discharges produced in either hydrogen or deuterium [for which $\mu\left(\mathrm{H}_{3}^{+}\right) \simeq 1.3 \mu\left(\mathrm{D}_{3}^{+}\right)$, whereas $\sigma_{c}\left(\mathrm{H}_{2}\right) \simeq \sigma_{c}\left(\mathrm{D}_{2}\right)$ [5]) both exhibit DIS [1], hence confirming the idea that this phenomenon depends on more than just ion mobility values.

In order to analyze the conditions responsible for the formation of DIS, simulations of CCRF discharges, running under similar operating conditions, were carried out in hydrogen, helium and in modified versions of these gases (obtained by assuming different mobility values either for electrons or for their ion species). Simulations used a fluid model describing the dynamics of charged particles in CCRF discharges [3]-[4], which was applied to a GEC reference Cell [4] with standard radial dimension $0 \leq r \leq 6.4 \mathrm{~cm}$ and axial dimension $0 \leq z \leq 3.2 \mathrm{~cm}$, where a lateral grounded grid was used to confine the plasma between the electrodes.

Figs. 1(a) and 2(a) plot the space-time variation with the Ionization Rate (IR) of hydrogen and helium, respectively, obtained at $r=0$ and during one RF period, for $13.56 \mathrm{MHz}$ frequency, $217 \mathrm{~V}$ applied RF voltage and 1 torr pressure. For comparison purposes, Figs. 1(b), 2(b) and 2(c) plot the IR, obtained under the same work conditions as before, for the following modified versions of hydrogen and helium: $\mu\left(\mathrm{H}_{3}^{+}\right) / 10$ [Fig. 1(b)]; $5 \mu\left(\mathrm{He}^{+}\right)$[Fig. 2(b)]; and $3 \sigma_{c}(\mathrm{He})$ [Fig. 2(c)]. Results for hydrogen show the development of four peaks associated to the formation of DIS, which are not present in helium discharges [note also the different color scale between 
Figs. 1 and 2].

The formation of these peaks can be explained as follows. In gases like hydrogen, featuring a high ion-to-electron mobility ratio, the contraction of a space-charge sheath is accompanied by a compression of positive ions toward the electrode and an accumulation of electrons at the plasma-sheath border. Under these circumstances, the conventional (outward) space-charge field is followed by an (inward) electric field (directed from the positive sheath to the negative plasma-sheath border), and by a second (outward) electric field, which acts as an extra sheath barrier to limit the electron flux. This inward-to-outward field inversion phenomenon explains the formation of double sheaths in RF discharges [3]. Consequently, at the beginning and at the end of the RF period, electrons at sheath border are accelerated toward the RF electrode by the inward electric field. Some of these electrons will acquire enough energy to ionize the background gas, thus producing the first peaks located at $z \simeq 0.4 \mathrm{~cm}$ and $t \simeq 0$ or $t \simeq 70-74 \mathrm{~ns}$. At a later time, electrons located at the RF sheath entrance are heated by the increasing electric field in this region and, while the sheath expands, they are able to produce a second ionization peak (more intense than the first one), located at $z \simeq 0.6 \mathrm{~cm}$ and $t \simeq 28 \mathrm{~ns}$. Simultaneously with the increase in the RF electric field, a field inversion phenomenon occurs in the opposite sheath. Thus, as we approach half period, electrons are accelerated toward the grounded electrode and a third ionization peak is observed at $z \simeq 2.9 \mathrm{~cm}$ and $t \simeq 29 \mathrm{~ns}$. Later on (after $t=37 \mathrm{ns)}$, and similarly to what happens within the RF sheath, the electron population very close to the grounded electrode is subject to Joule heating, due to an increase in the electric field in this region. Thus, at $z \simeq 2.7 \mathrm{~cm}$ and $t \simeq 65 \mathrm{ns,} \mathrm{a} \mathrm{fourth} \mathrm{ionization} \mathrm{peak} \mathrm{is} \mathrm{observed,} \mathrm{as} \mathrm{the} \mathrm{grounded}$ sheath expands.

An observation of Fig. 1 reveals that, in hydrogen, DIS are attenuated if $\mu\left(\mathrm{H}_{3}^{+}\right)$is strongly reduced (this also explains why a reduction of $\mu\left(\mathrm{H}_{3}^{+}\right)$by a factor of only 1.3 , like with deuterium, does not produce significant changes in DIS formation). Conversely, Fig. 2 shows that, in helium, the formation of DIS is enhanced by an increase in either $\mu\left(\mathrm{He}^{+}\right)$or $\sigma_{c}(\mathrm{He})$ (i.e., a decrease in the electron mobility). In conclusion, results show that the development of DIS is neither exclusive of gases featuring high-mobility ions, nor is only due to high electron-neutral collision cross-sections, being favored instead by high ion-to-electron mobility ratios. 


\section{REFERENCES}

[1] W. G. Graham and C. M. O. Mahoney, "Experimental studies of rf sheath" in Electron Kinetics and Applications of Glow Discharges. ser. NATO ASI, U. Kortshagen and L. D. Tsendin, Eds. New York: Plenum, 1998, vol. 367, pp. 503-510.

[2] O. Leroy, P. Stratil, J. Perrin, J. Jolly and P. Belenguer, "Spatiotemporal analysis of the double layer formation in hydrogen radio frequency discharges,” J. Phys. D: Appl. Phys., vol. 28, pp. 500-507, 1995.

[3] A. Salabas, L. Marques, J. Jolly, G. Gousset and L. L. Alves, "Systematic characterization of low-pressure capacitively coupled hydrogen discharges," J. Appl. Phys., vol. 95, pp. 4605-4620, 2004.

[4] A. Salabas, G. Gousset and L. L. Alves, "Two-dimensional fluid modeling of charged particle transport in radio-frequency capacitively coupled discharges,” Plasma Sources Sci. Technol., vol. 11, pp. 448-465, 2002.

[5] E. W. McDaniel, Collision Phenomena in Ionized Gases. New York: Wiley, 1964, chs. 9 and 11. 


\section{Figure captions}

Fig. 1. Space-time variation (at $r=0$ and during one RF cycle) with the ionization rate (in $10^{14} \mathrm{~cm}^{-3} \mathrm{~s}^{-1}$ ), for a CCRF discharge operating at $13.56 \mathrm{MHz}$ frequency, $217 \mathrm{~V}$ applied voltage and 1 torr pressure, in (a) standard hydrogen; (b) modified hydrogen adopting $\mu\left(\mathrm{H}_{3}^{+}\right) / 10$.

Fig. 2. As in Fig. 1, for (a) standard helium; (b) modified helium adopting $5 \mu\left(\mathrm{He}^{+}\right)$; (c) modified helium adopting $3 \sigma_{c}(\mathrm{He})$. 

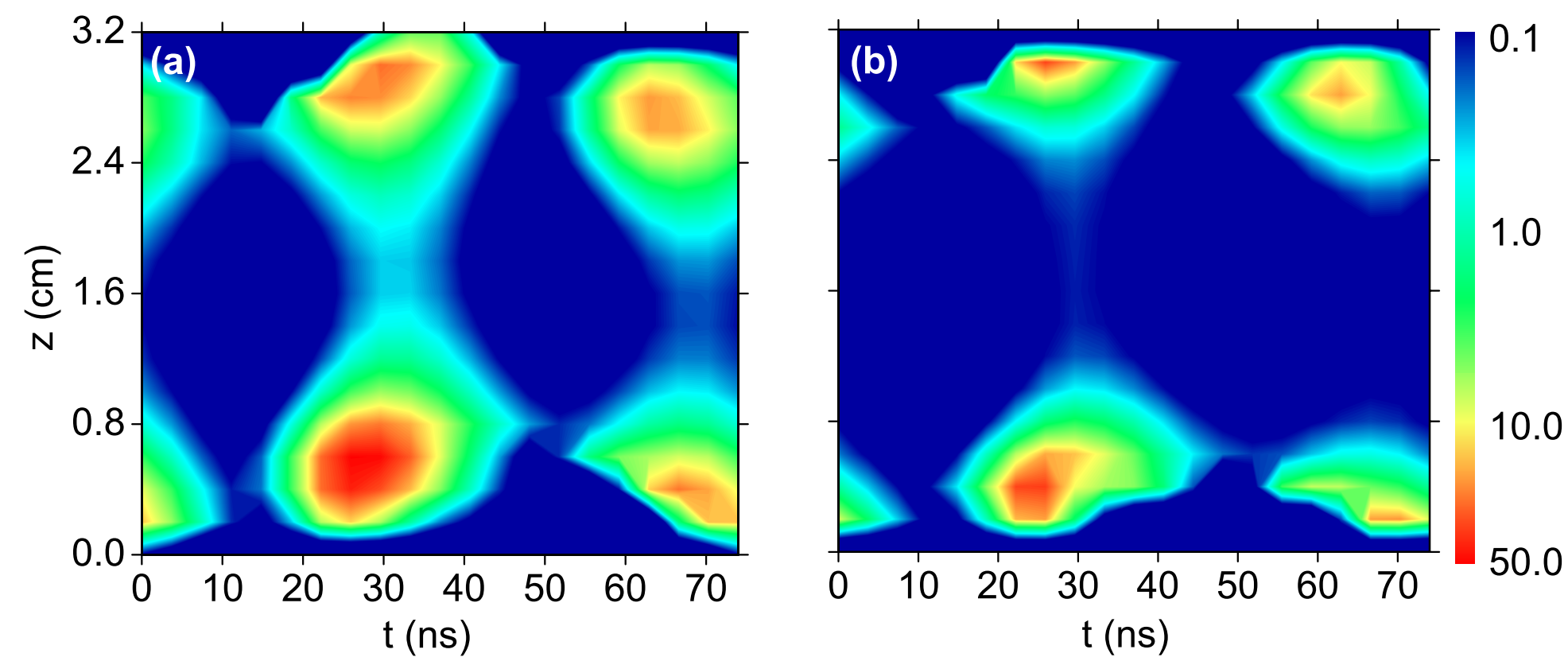

Figure 1. 
